\title{
$\angle$ Research Square

\section{Distance between the center of the foveal avascular zone measured automatically and the highest foveal bulge using OCT angiography}

Takuhei Shoji ( $\nabla$ shoojii@gmail.com )

Saitama Medical University

Hirokazu Ishii

Saitama Medical University

Junji Kanno

Saitama Medical University

Takanori Sasaki

Saitama Medical University

Yuji Yoshikawa

Saitama Medical University

Hisashi lbuki

Saitama Medical University

Kei Shinoda

Saitama Medical University

\section{Research Article}

Keywords: fovea, foveola, cone photoreceptors, foveal avascular zone (FAZ)

Posted Date: June 24th, 2021

DOl: https://doi.org/10.21203/rs.3.rs-646634/v1

License: (c) (1) This work is licensed under a Creative Commons Attribution 4.0 International License.

Read Full License 


\section{Abstract}

The center of the fovea, termed the foveola, is the area of highest visual acuity, has the highest density of cone photoreceptors, and is the center of the foveal avascular zone (FAZ). We investigated the distance between the automatically-determined center of the foveal avascular zone (FAZ) and the manuallydetermined highest foveal bulge (FB) point using swept-source optical coherence tomography angiography (OCTA). This cross-sectional study included 49 eyes of 49 individuals ( 34 women and 15 men; median age: 68 years) with no history of ocular disorders. The FAZ in the superficial capillary plexus was automatically determined using the Kanno-Saitama Macro method, and the center of the FAZ was automatically determined using ellipse approximation. The foveal center was manually identified as the highest FB point on B-scan OCTA images. The center of the FAZ was more likely to be located inferior to the highest FB point. In participants with a combined distance of more than $50 \mu \mathrm{m}$ between the center of the FAZ and the highest FB point, the displacement was significantly more in the horizontal direction than in the vertical direction $(p=0.017)$. These results can be applicable to further studies regarding the spatial relationships between the center of the FAZ and the highest FB point in various macular diseases or previously-treated eyes.

\section{Introduction}

The center of the fovea, termed the foveola, is the area of highest visual acuity, has the highest density of cone photoreceptors and the smallest thickness of the fovea, and is the center of the foveal avascular zone (FAZ). ${ }^{1}$ Optical coherence tomography (OCT) is a non-invasive imaging technology used to acquire high-resolution, 3D, cross-sectional images of the retina, and is one of the most important ancillary tools for the diagnosis and management of macular diseases. ${ }^{2,3}$ Both spectral-domain OCT (SD-OCT) and swept-source OCT (SS-OCT) provide detailed and in vivo analyses of the interior of the retina, especially the fovea, which is the area with the highest visual acuity. The foveal center is also a key reference landmark of the retina, as the early treatment diabetic retinopathy study (ETDRS) grid generated from the foveal center is an important tool for doctors to diagnose retinal diseases. ${ }^{4}$ Therefore, the accurate identification of the foveal center is of great significance for disease evaluation and diagnosis.

The foveal bulge (FB) is the bulge at the center of the fovea observed on $\mathrm{OCT}^{5,6}$ and studies of crosssectional OCT images of healthy eyes have reported that the ellipsoid zone has a bulge at the central fovea. ${ }^{5,6}$ The FB is defined as a bulge in the EZ at the center of the fovea. ${ }^{5-7}$ The integrity of the foveal microstructure has been used to assess the function of the fovea in various retinal diseases. The presence or absence of the FB has also been shown to be significantly correlated with visual acuity in various retinal disorders. $5,8,9$

The foveal vascular network is composed of interconnected capillaries that perfuse the inner retinal layer. This network forms a ring at the margin of the fovea and produces a capillary-free region referred to as the FAZ. ${ }^{10}$ The FAZ is highly sensitive to ischemic events and has been implicated in several pathological processes. Previous studies have reported enlarged FAZs in retinal ischemic diseases (including diabetic 
retinopathy $)^{11}$ and retinal vascular obstruction. ${ }^{12}$ In addition, the FAZ has been evaluated in glaucoma, ${ }^{13,1415}$ and idiopathic epiretinal membrane (ERM). ${ }^{16}$ In recent reports, the center of fovea pit, center of the FB, and center of the FAZ have been located manually using OCT angiography (OCTA), though the manually-located landmarks are not always accurate. ${ }^{16,17}$ Recently, we generated and reported a macro program to automatically determine the FAZ area using OCTA. ${ }^{15,18}$

Although previous studies have suggested that both the FAZ and FB are critical biomarkers of the functional properties of the fovea in eyes with various retinal diseases, the spatial relationship between the center of the FAZ in en-face images and the highest FB point are poorly understood. Therefore, this study investigated the positional relationship between the OCT-derived center of FAZ and the highest FB point in healthy eyes using OCTA images and an automated FAZ extraction program.

\section{Results}

This study included 49 eyes from 49 healthy participants. The participants' ocular characteristics are presented in Table 1. The median participant age was 68 years (interquartile range (IQR): 67-72 years), and 34 participants $(69.4 \%)$ were women. The decimal best-corrected visual acuity (BCVA) values were converted to logarithm of the minimum angle of resolution (logMAR) values. The median logMAR was -0.08 (IQR: -0.08- -0.08), axial length was $23.3 \mathrm{~mm}$ (IQR: 22.8-23.9 mm), spherical equivalent was -0.00 diopters (IQR: -0.38-1.25 diopters), and intraocular pressure (IOP) was $14.3 \mathrm{mmHg}$ (IQR: $12.3-16.3 \mathrm{mmHg}$ ) Figure 1 shows the direction and distance of the eccentric position of the center of the FAZ from the highest FB point. Figure 2 shows the distribution of the distance from the highest FB point to the center of the FAZ in the vertical (upper panel) and horizontal directions (lower panel), and a summary is shown in Table 2. The distribution of the distance in the vertical direction is mostly inferior to the highest FB point, while the distribution in the horizontal direction is close to the highest FB point (Figure 2). In the horizontal direction, the number of participants with a nasal- or temporal-shifted center of the FAZ were 26 and 23, respectively. Overall, the median horizontal shift of the center of the FAZ was $2.0 \mu \mathrm{m}$ in the nasal direction from the highest FB point. In the vertical direction, the center of the FAZ was more likely to be located inferior to the highest FB point (32 of 49 eyes, 65.3\%), with a median distance of $10.5 \mu \mathrm{m}$ from the highest FB point, which was significantly different from the reference value of $\pm 0(p=0.031$, Wilcoxon signed-rank test).

\section{Table 1. Participant characteristics}




\begin{tabular}{lc}
\hline No. of patients $(\mathrm{n})$ & 49 \\
\hline Age (years) & $68(67,72)$ \\
\hline Gender (Male / Female) & $15 / 34$ \\
\hline BCVA (Log MAR) & $-0.08(-0.08,-0.08)$ \\
\hline SE (Diopters) & $0.00(-0.38,1.25)$ \\
\hline Axial Length (mm) & $23.3(22.8,23.9)$ \\
\hline IOP (mmHg) & $14.3(12.3,16.3)$ \\
\hline
\end{tabular}

Data are presented as numbers or median (interquartile range)

Abbreviations: LogMAR, logarithm of the minimum angle of resolution SE, Spherical equivalent; IOP, intra ocular pressure

LogMAR represents the log of the decimal best corrected visual acuity

Table 2. Distance to the center of the foveal avascular zone from the highest foveal bulge point

\begin{tabular}{lcc}
\hline & Direction and distance & P \\
\hline Horizontal Direction & $26 / 23$ & \\
Nasal / Temporal $(\mathrm{n})$ & $2.0(-26.2,23.5)$ (nasal) & 0.914 \\
Distance $(\mu \mathrm{m})$ & $17 / 32$ & \\
Vertical Direction & & \\
Superior/ Inferior $(\mathrm{n})$ & $0.5(-9.0,26.3)$ (inferior) & 0.031 \\
\hline Distance $(\mu \mathrm{m})$ &
\end{tabular}

Data are presented as numbers or median (interquartile range)

*P values obtained using a one-sample Wilcoxson signed rank test with a reference value of zero

Figure 3 and Table 3 show the direction and distribution of the total distance from the highest FB point to the center of the FAZ. In 31 eyes (63.2\%), the total distance was less than $50 \mu \mathrm{m}$. In the eyes with a total distance of more than $50 \mu \mathrm{m}(\mathrm{n}=18)$, the distance was significantly greater in the horizontal direction than in the vertical direction ( $p=0.017$, Wilcoxon signed-rank test).

Table 3. Total distance between the center of the FAZ and the highest point of the foveal bulge

\begin{tabular}{cccccc}
\hline Distance & $\mathrm{n}$ & Total Distance $(\mu \mathrm{m})$ & Horizontal $(\mu \mathrm{m})$ & Vertical $(\mu \mathrm{m})$ & $\mathrm{P} *$ \\
\hline Overall & 49 & $39.7(23.4-69.5)$ & $24.0(9.8-53.0)$ & $21.5(9.5-31.5)$ & 0.053 \\
$<50 \mu \mathrm{m}$ & 31 & $24.8(18.1-37.9)$ & $14.0(4.5-26.5)$ & $14.5(6.5-26.0)$ & 0.931 \\
$\geqq 50 \mu \mathrm{m}$ & \multirow{2}{*}{18} & $78.3(61.6-87.3)$ & $68.5(46.5-79.0)$ & $28.5(23.0-49.8)$ & 0.014 \\
\hline
\end{tabular}

Data are presented as numbers or median (interquartile range)

*P values obtained using a Wilcoxon signed-rank test

\section{Discussion}


This study evaluated the spatial relationship between the automatically-determined center of the OCTAderived FAZ and the center of the highest FB point. The distance between the center of the FAZ and the highest FB point was generally close but was not aligned in healthy eyes, with an average distance of approximately $30 \mu \mathrm{m}$ between the two points. While the positions of the center of the FAZ and the highest FB point were similar in the horizontal direction, the center of the FAZ was located significantly inferior to the highest FB point in the vertical direction. In participants with a combined distance of more than $50 \mu \mathrm{m}$ between the center of the FAZ and the highest FB point, the displacement was significantly more in the horizontal direction than in the vertical direction.

In this study, the FAZ was identified automatically using the Kanno-Saitama Macro (KSM) method. ${ }^{18}$ The program utilizing the OCTA instruments faced several limitations, and required manual delineation of FAZ, unlike in this study. ${ }^{16}$ The automated methods are more objective and reproducible than the manual method, and the specific KSM method and its clinical applications have been reported. ${ }^{15,18}$ The identification of the FAZ has excellent reproducibility and is comparable to measurements obtained manually. Thus, it is easy to measure the precise distances with high reproducibility. The precise spatial relationship between the center of the FAZ and the highest FB point is clinically relevant as the macular region, including the fovea, is closely associated with visual prognosis. An understanding of the relationships between the highest FB point and the FP is necessary to investigate the significance of the location of the center of the FAZ.

Prior to the widespread use of OCTA, the positional relationship between the center of the FAZ and the highest FB point was poorly understood as FAZ measurements using fluorescein angiography were not routine, especially in healthy individuals. Furthermore, the ability to accurately compare and measure the distance between the center of the FAZ and the highest FB point was made possible with the development of OCTA technology, which provides both in vivo en-face images and B scan images of the macular region as digital images. Several recent studies have reported that the FB, foveal pit (FP), and center of the FAZ, which are considered to indicate the center of the central fovea, are misaligned even in healthy eyes. ${ }^{16,17,19}$ Kuppuswamy et al. reported that the position of the FB nasally deviates from the FP in healthy eyes, with a median distance of $58.6 \mu \mathrm{m} .{ }^{19}$ Matsui et al. reported that the highest FB point was on the nasal side of the FP in 97 eyes (66\%) and the superior side of the FP in 82 eyes (55\%) in a study of young, healthy, Japanese participants. ${ }^{17}$ The results of the previous study are consistent with the results of this study regarding the position of the highest FB point in relation to the center of the FAZ. However, this study found no significant difference between the position of the highest FB point and the center of the FAZ in the horizontal direction. Hasegawa et al. reported the degree of misalignment between the center of the FAZ and the highest FB point in individuals with ERM in one eye and a healthy contralateral eye. ${ }^{16}$ The mean distance from the highest FB point to the center of the FAZ was $38.7 \pm 20.0 \mu \mathrm{m}$ in the healthy eyes, which is consistent with the results of the current study. The previous study reported that the degree of misalignment between the center of the FAZ and the highest FB point was greater in eyes with more advanced ERM, which may be useful for evaluating the degree of ERM traction. However, the FB and FP can only be identified manually, even with the use of current technology. ${ }^{17,19}$ Moreover, it 
remains unclear whether the highest FB point or the FP contributes more to visual acuity. Future studies regarding various retinal diseases, such as rhegmatogenous retinal detachment and retinal vein occlusion, should be conducted to determine the positional relationship between the center of the FAZ and the highest FB point and the pathophysiology of various retinal diseases.

In this study, eyes with a total distance of more than $50 \mu$ m between the center of the FAZ and the highest FB point had significantly greater misalignment in the horizontal direction than in the vertical direction. A recent study reported that foveal misalignment was greater in eyes with ERM with ectopic inner foveal layers (EIFLs) than in those without EIFLs, especially in the horizontal direction, with an average distance of more than $100 \mu \mathrm{m} .{ }^{16}$ As the participants in this study were elderly individuals with healthy eyes, subclinical changes may have occurred in the eyes with greater foveal misalignment. Although these relationships are unclear due to the limitations of cross-sectional studies, the pathogenesis and progression of ERM should be studied in longitudinal studies in the future.

This study has several limitations. First, the age distribution of the participants in this study was not sufficiently wide, and the number of participants was not large. A previous report suggested that FB was more commonly observed in younger individuals. ${ }^{20}$ However, age is not a specific confounder of this study as it compares the distance between the OCT-derived center of the FAZ and the highest FB point according to the imaging setting in the same participant and does not compare the misalignment between age groups. Although there are no indications that this observation will not apply to individuals of different ages, future studies to confirm the distance in younger individuals are needed. Second, although the center of the FAZ was more likely to be located inferior to the highest FB point and was significantly different compared to a reference value of \pm 0 , the difference was relatively small $(10 \mu \mathrm{m})$. Thus, even though the results showed statistical significance, they may not be clinically meaningful. However, the purpose of this study was to investigate the distance between the center of the automatically-determined, OCTA-derived FAZ area and the manually-determined highest FB point. The results of this study indicate that the center of the FAZ and the highest FB point are near one another in healthy individuals. These results will improve the understanding of diseased and treated eyes.

In conclusion, the distance between the center of the automatically-determined, OCTA-derived FAZ area and manually-determined highest FB point. is minimal, but was not aligned in elderly participants with healthy eyes. These results can be applicable to further studies regarding the spatial relationships between the center of the FAZ and the highest FB point in various macular diseases or previously-treated eyes.

\section{Methods}

\section{Study population}

This cross-sectional study of healthy subjects was approved by the Ethics Committee of Saitama Medical University (No. 20013.01) and was conducted in accordance with the tenets of the Declaration of 
Helsinki. Healthy participants that were 20 years of age or older who fulfilled the eligibility requirements were enrolled in the study. All participants provided informed consent. The study period was October 2017 to November 2017.

Healthy participants were recruited from the ophthalmology outpatient clinic of Saitama Medical University Hospital (Saitama, Japan), except for refractive error. All participants underwent a comprehensive ophthalmic examination, including slit lamp biomicroscopy, IOP measurement via noncontact tonometry (Tonoref II, Nidek Co., Ltd., Aichi, Japan), and fundus photography (CX-1, Canon Inc., Tokyo, Japan). The axial length and CCT were measured using an optical biometer (OA-2000, Tomey Corp., Nagoya, Japan). Horizontal and vertical B-scan images and en-face images of the area around the macula were obtained using swept-source optical coherence tomography angiography (SS-OCTA; PlexElite9000 Carl Zeiss Meditec, Jena, Germany).

Participants $<20$ years of age with a reflective error $>+3.0$ diopters or $<-6.0$ diopters, an axial length $>26$ $\mathrm{mm}$, other ocular diseases, diabetic retinopathy, retinal vein/artery occlusion, age-related macular degeneration, retinal detachment, tilted disc, exfoliation syndrome, high myopia, ocular neuropathy without mild ametropia, or a history of ocular surgery (except uncomplicated cataract surgery) were excluded from the study. Participants in whom images of poor quality were obtained (signal strength $<8$ due to signal noise; 1 = minimum, $10=$ maximum) were also excluded from the study.

\section{Optical coherence tomography angiography}

A $3 \times 3 \mathrm{~mm}(1,024 \times 1,024$ pixels $)$ OCTA image centered on the fovea was scanned using SS-OCTA using a central wavelength of 1,060 nm, an A-scan rate of 100,000 scans per second, an axial resolution of approximately $5 \mu \mathrm{m}$ in tissue, and an estimated lateral resolution at the retinal surface of approximately $12 \mu \mathrm{m} .{ }^{21}$ The angiography image was processed using phase/Doppler shift and amplitude variation (Optical Micro-Angiography). ${ }^{22}$ The scan contained 300 A-lines $\times 300$ locations with four repeated scans in each fixed location.

\section{Measurements of the center of the FAZ using OCTA en-face images}

The area of the FAZ $\left(\mathrm{mm}^{2}\right)$ was calculated using ImageJ and an original macro language (KSM program), as previously described. ${ }^{18}$ In brief, the SS-OCTA software generates en-face images from slabs at different layers via automated segmentation. The superficial retinal layer (SRL) was used to measure the area of the FAZ. The SRL was defined as the area between the internal limiting membrane layer and the inner plexiform layer. KSM is an automated analysis program that extracts the area of the FAZ. The FAZ was defined as the area denoted by the connected points along the borders of the identifiable capillary network in the parafoveal area. The extracted FAZ showed excellent reproducibility and was comparable to manual measurements. ${ }^{18}$ After identifying the FAZ, the center of the FAZ was automatically identified as the center of an elliptic approximation using the ImageJ program (Figure 4).

\section{Measurements of the height of the FB}


The height of the FB was measured as the highest FB point, as previously reported. ${ }^{16,17}$ The height of the FB was defined as the distance between the outer boundary of the ellipsoid zone (EZ) and the inner boundary of the Bruch's membrane at the vertex of the FB (Figure 25). A single observer (HI) who was blinded to the participants' characteristics measured the highest FB points in both the horizontal and vertical B scans in duplicate. The intersection of the highest FBs in the horizontal and vertical scans was defined as the highest FB point. The final highest FB point was the center point of each measurement of the highest FB point. The distance between the central point of the FAZ and the highest FB point was calculated (Figure 6). The magnification effect was adjusted due to axial length according to the manufacturer's correction formula, and it was confirmed that the correction was comparable to that in a previous study. ${ }^{23}$

\section{Statistical analysis}

The distribution of numerical variables was assessed via the inspection of histograms and the use of the Shapiro-Wilk W test for normality. Normally-distributed variables are reported as mean \pm standard deviation. Non-normally distributed variables are reported as median (IQR). The horizontal and vertical positions of the highest FP points from the center of the FAZ were determined using a one-sample Wilcoxon signed rank test with \pm 0 as the reference point. The Wilcoxon signed-rank test was used to compare the horizontal and vertical distances. Statistical significance was set at $\mathrm{P}<0.05$. All statistical analyses were conducted using JMP version 10.1 software (SAS Institute Inc., Cary, NC, USA) and Stata software version 15 (StataCorp LP, College Station, TX, USA).

\section{Declarations}

\section{Acknowledgments}

\section{Author Contributions Statement}

Designed and conducted the study: TSh, HI, KS, and KS. Data collection: TSh, HI, JK, TSa, and YY. Data analysis and interpretation: TSh and HI. Writing: TSh. Critical revision: IH and SK. Manuscript approval: TSh, HI, JK, TSa, YY, and KS.

\section{Competing interests}

The authors declare no competing interests.

\section{Funding support}

This work was supported by a Grant-in-Aid for Young Researchers at Saitama Medical University Hospital (Saitama, Japan) and a grant from the Japan Society for the Promotion of Science (KAKENHI Grant Number 19K09976).

\section{Data availability}


The datasets generated and/or analyzed during the current study are available from the corresponding author upon reasonable request.

\section{References}

1 Bringmann, A. et al. The primate fovea: Structure, function and development. Prog Retin Eye Res 66, 49-84, doi:10.1016/j.preteyeres.2018.03.006 (2018).

2 Drexler, W. \& Fujimoto, J. G. State-of-the-art retinal optical coherence tomography. Prog Retin Eye Res 27, 45-88, doi:10.1016/j.preteyeres.2007.07.005 (2008).

3 Geitzenauer, W., Hitzenberger, C. K. \& Schmidt-Erfurth, U. M. Retinal optical coherence tomography: past, present and future perspectives. Br J Ophthalmo/ 95, 171-177, doi:10.1136/bjo.2010.182170 (2011).

4 Massin, P. et al. Retinal thickness in healthy and diabetic subjects measured using optical coherence tomography mapping software. Eur J Ophthalmo/ 12, 102-108, doi:10.1177/112067210201200205 (2002).

5 Hasegawa, T., Ueda, T., Okamoto, M. \& Ogata, N. Relationship between presence of foveal bulge in optical coherence tomographic images and visual acuity after rhegmatogenous retinal detachment repair. Retina 34, 1848-1853, doi:10.1097/IAE.0000000000000160 (2014).

6 Hasegawa, T., Ueda, T., Okamoto, M. \& Ogata, N. Presence of foveal bulge in optical coherence tomographic images in eyes with macular edema associated with branch retinal vein occlusion. $A m \mathrm{~J}$ Ophthalmo/ 157, 390-396 e391, doi:10.1016/j.ajo.2013.10.007 (2014).

7 Watanabe, K., Tsunoda, K., Mizuno, Y., Akiyama, K. \& Noda, T. Outer retinal morphology and visual function in patients with idiopathic epiretinal membrane. JAMA Ophthalmo/ 131, 172-177, doi:10.1001/jamaophthalmol.2013.686 (2013).

8 Chen, C. J. et al. Characterizing the phenotype and genotype of a family with occult macular dystrophy. Arch Ophthalmol 130, 1554-1559, doi:10.1001/archophthalmol.2012.2683 (2012).

9 Al-Haddad, C. E., El Mollayess, G. M., Mahfoud, Z. R., Jaafar, D. F. \& Bashshur, Z. F. Macular ultrastructural features in amblyopia using high-definition optical coherence tomography. $\mathrm{Br} J$ Ophthalmo/ 97, 318-322, doi:10.1136/bjophthalmol-2012-302434 (2013).

10 Chui, T. Y., VanNasdale, D. A., Elsner, A. E. \& Burns, S. A. The association between the foveal avascular zone and retinal thickness. Invest Ophthalmol Vis Sci 55, 6870-6877, doi:10.1167/iovs.1415446 (2014).

11 Tang, F. Y. et al. Determinants of Quantitative Optical Coherence Tomography Angiography Metrics in Patients with Diabetes. Sci Rep 7, 2575, doi:10.1038/s41598-017-02767-0 (2017). 
12 Koulisis, N. et al. Quantitative microvascular analysis of retinal venous occlusions by spectral domain optical coherence tomography angiography. PLoS One 12, e0176404, doi:10.1371/journal.pone.0176404 (2017).

13 Choi, J. et al. Quantitative optical coherence tomography angiography of macular vascular structure and foveal avascular zone in glaucoma. PLoS One 12, e0184948, doi:10.1371/journal.pone.0184948 (2017).

14 Kwon, J., Choi, J., Shin, J. W., Lee, J. \& Kook, M. S. Glaucoma Diagnostic Capabilities of Foveal Avascular Zone Parameters Using Optical Coherence Tomography Angiography According to Visual Field Defect Location. J Glaucoma 26, 1120-1129, doi:10.1097/IJG.0000000000000800 (2017).

15 Shoji, T. et al. OCT angiography measured changes in the foveal avascular zone area after glaucoma surgery. Br J Ophthalmol, doi:10.1136/bjophthalmol-2020-317038 (2020).

16 Hasegawa, T., Kawaguchi, A., Arakawa, H., Maruko, I. \& lida, T. Misalignment between center of foveal avascular zone and center of foveal photoreceptors in eyes with idiopathic epiretinal membrane. Retina, doi:10.1097/IAE.0000000000003064 (2020).

17 Matsui, Y., Miyata, R., Uchiyama, E., Matsubara, H. \& Kondo, M. Misalignment of foveal pit and foveal bulge determined by ultrahigh-resolution SD-OCT in normal eyes. Graefes Arch Clin Exp Ophthalmol, doi:10.1007/s00417-020-04813-6 (2020).

18 Ishii, H. et al. Automated Measurement of the Foveal Avascular Zone in Swept-Source Optical Coherence Tomography Angiography Images. Trans/ Vis Sci Techno/ 8, 28, doi:10.1167/tvst.8.3.28 (2019).

19 Kuppuswamy Parthasarathy, M. \& Bhende, M. Deviation in the Position of Foveal Bulge from Foveal Center in Normal Subjects Measured Using Spectral-Domain OCT. Ophthalmol Retina 2, 337-342, doi:10.1016/j.oret.2017.08.002 (2018).

20 Saurabh, K. et al. Age-related Changes in the Foveal Bulge in Healthy Eyes. Middle East Afr J Ophthalmo/24, 48-50, doi:10.4103/meajo.MEAJO_347_16 (2017).

21 Zhang, Q. et al. A Novel Strategy for Quantifying Choriocapillaris Flow Voids Using Swept-Source OCT Angiography. Invest Ophthalmol Vis Sci 59, 203-211, doi:10.1167/iovs.17-22953 (2018).

22 Bojikian, K. D. et al. Optic Disc Perfusion in Primary Open Angle and Normal Tension Glaucoma Eyes Using Optical Coherence Tomography-Based Microangiography. PLoS One 11, e0154691, doi:10.1371/journal.pone.0154691 (2016).

23 Moghimi, S. et al. Measurement of optic disc size and rim area with spectral-domain OCT and scanning laser ophthalmoscopy. Invest Ophthalmol Vis Sci 53, 4519-4530, doi:10.1167/iovs.11-8362 (2012). 
Figures

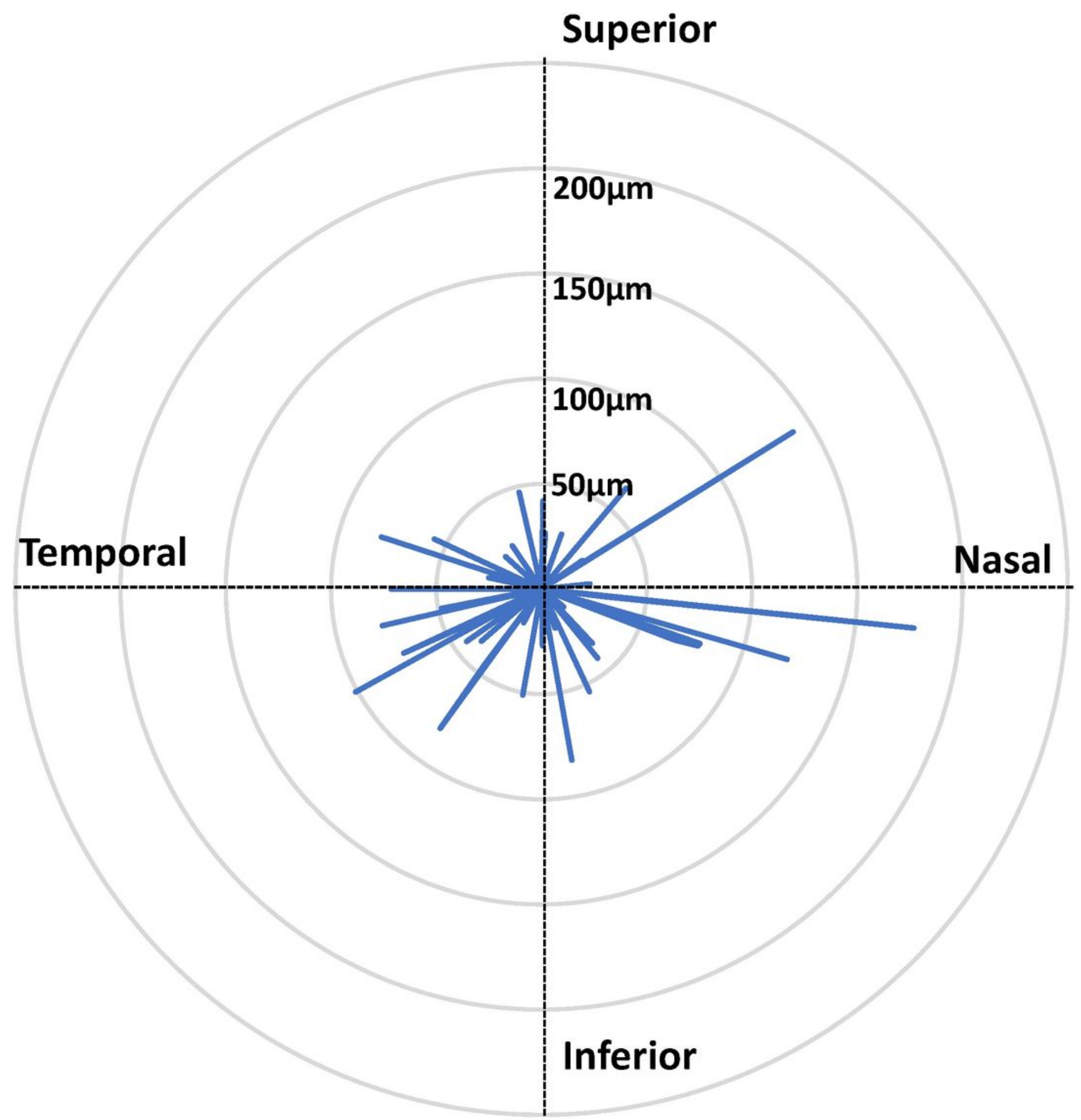

Figure 1

Direction and distance of the eccentric position of the center of the foveal avascular zone based on highest foveal bulge point 


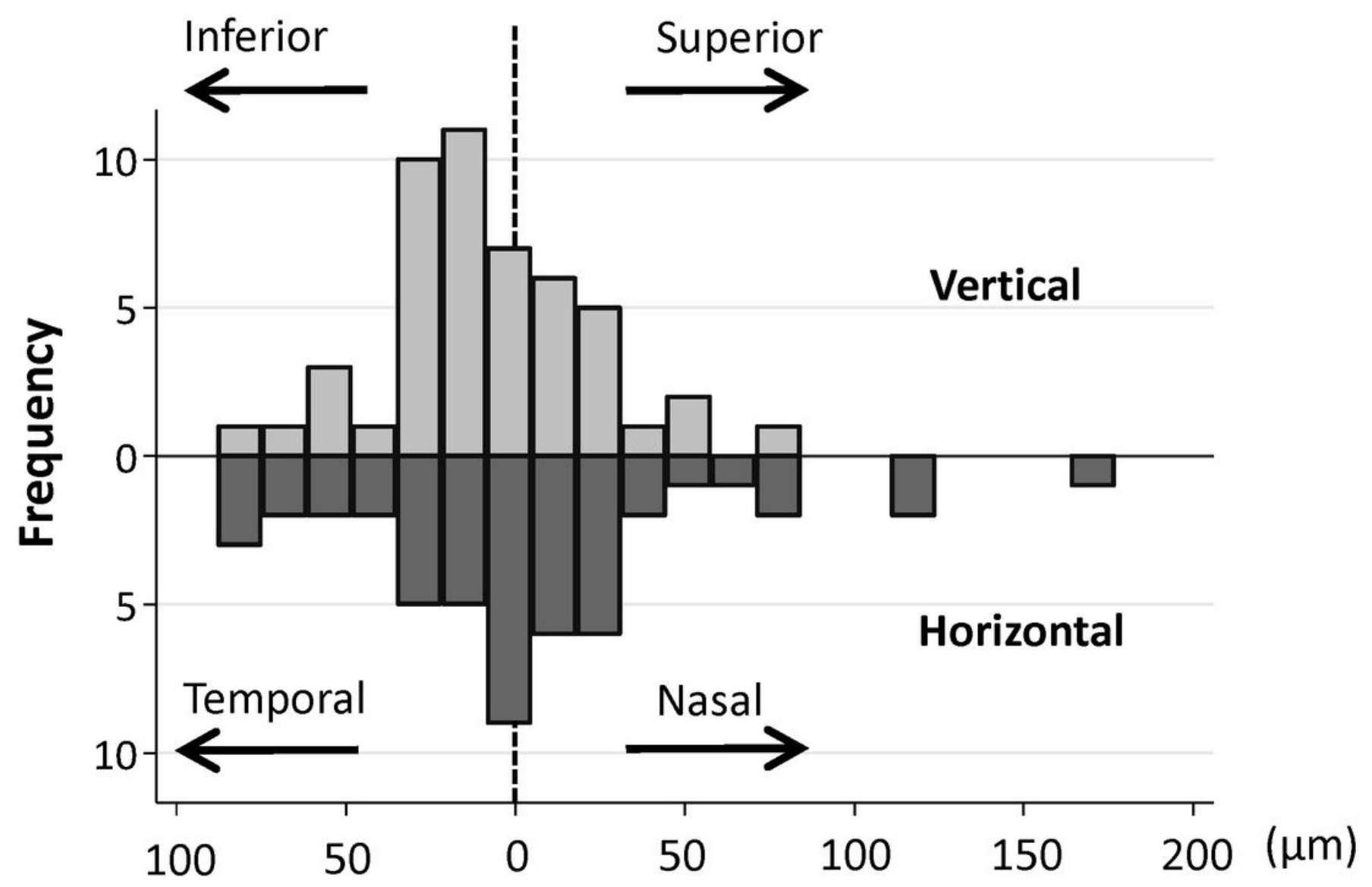

Figure 2

Distribution of the distance from the highest foveal bulge point to the center of the foveal avascular zone in the vertical and horizontal directions 

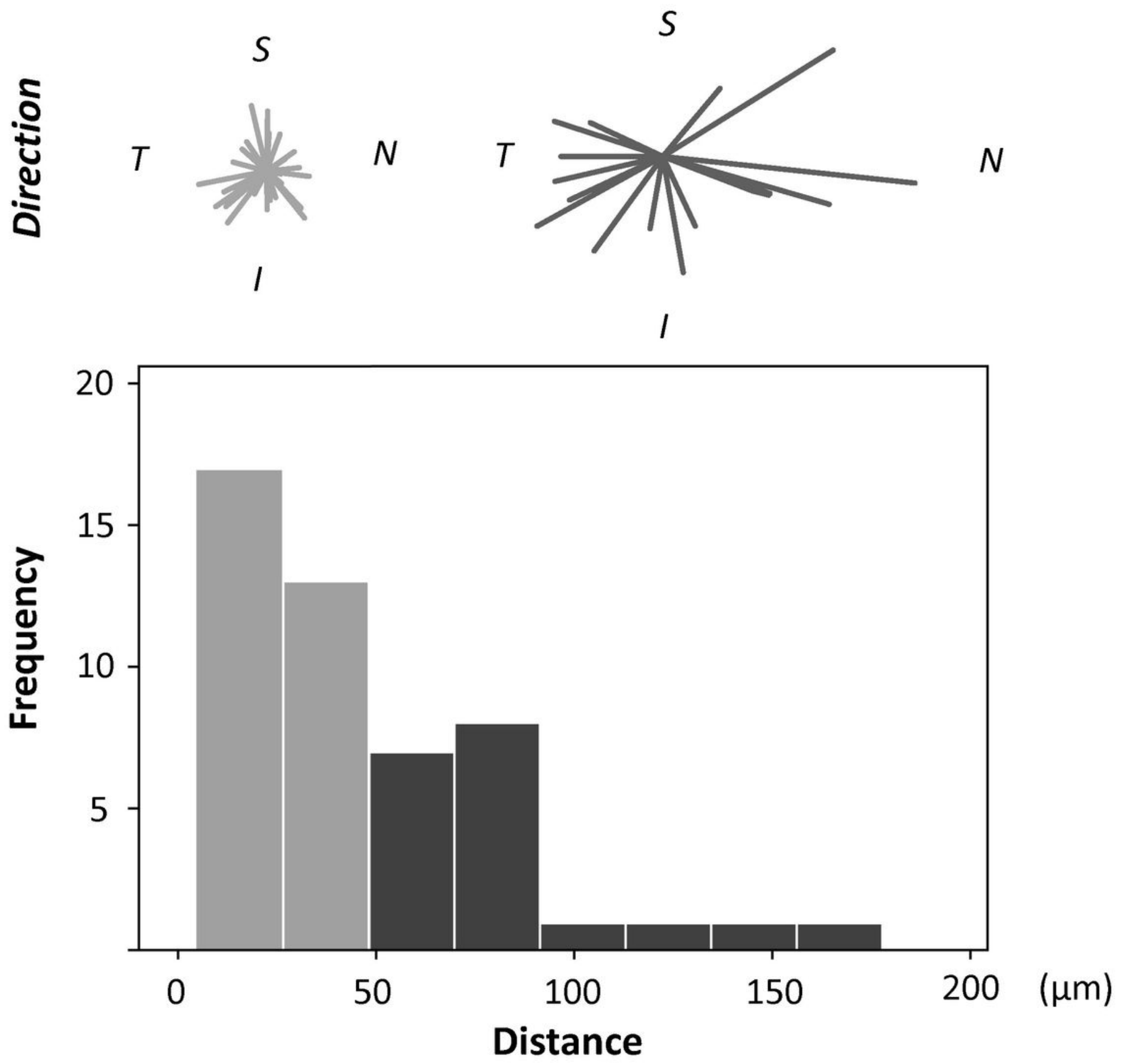

Figure 3

Total distance and direction of the highest foveal bulge point in relation to the center of the foveal avascular zone Light gray bars indicate eyes with distances less than $50 \mu \mathrm{m}$, and dark gray bars indicate distances equal or greater than $50 \mu \mathrm{m}$. 


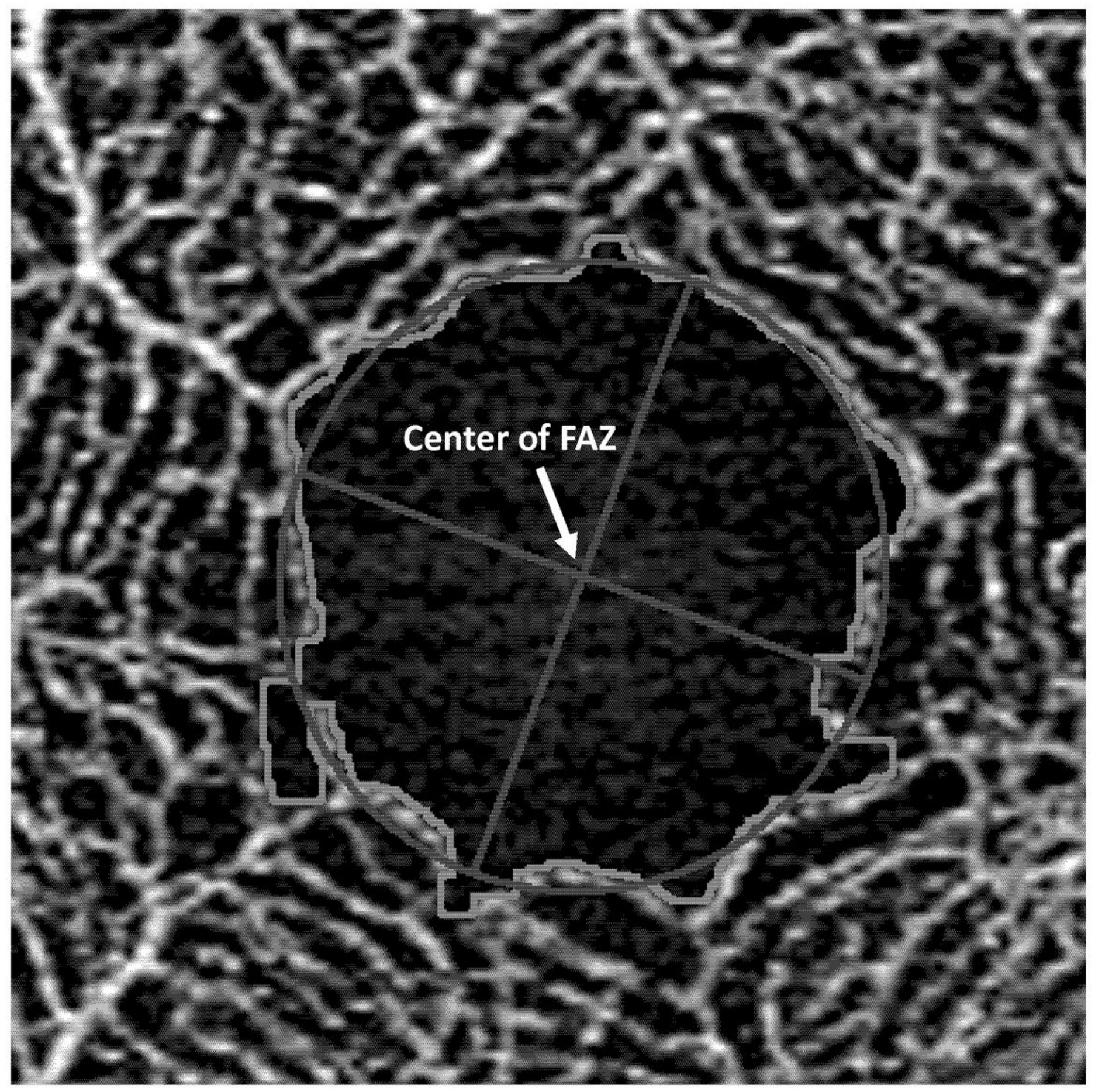

\section{Figure 4}

Identification of the center of the foveal avascular zone The central fossa vessel-free zone was extracted using the Kanno-Saitama Macro (KSM) method (red line). An approximate ellipse was created for the delineated foveal avascular zone (FAZ) (blue line). The center of the FAZ is defined as the intersection of the major and minor axes of the approximate ellipse. 


\section{Horizontal scan \\ (temporal to nasal)}

Vertical scan

(inferior to superior)

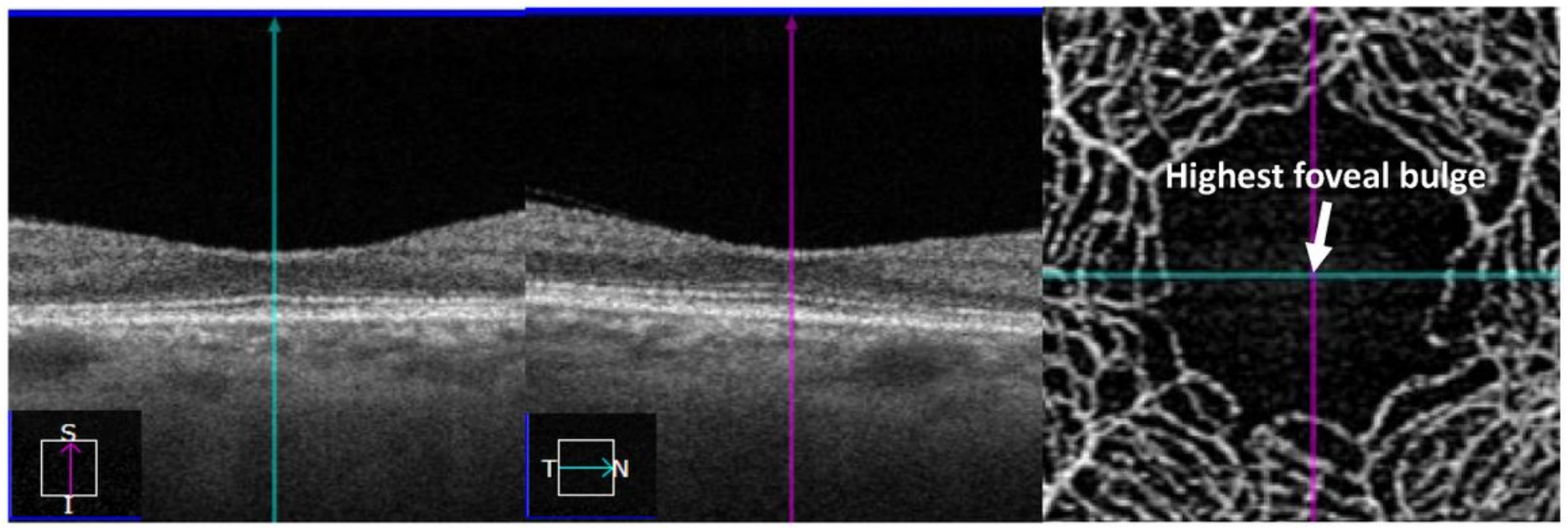

Figure 5

Identification of the highest foveal bulge point using optical coherence tomography angiography The intersection of the highest foveal bulge (FB) in the horizontal and vertical scans was defined as the highest FB point.

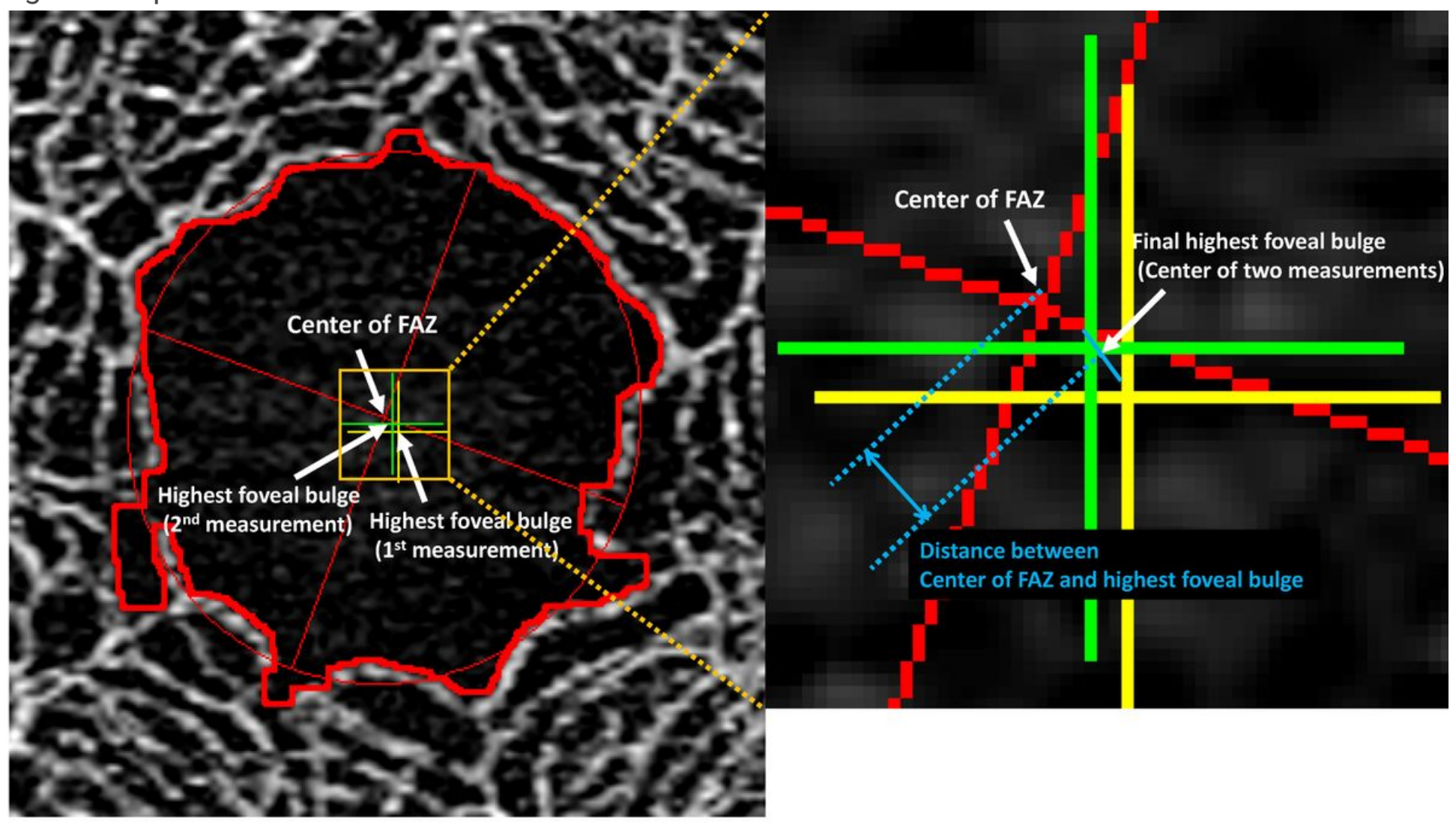

Figure 6 
Schematic explanation of the distance between the center of the foveal avascular zone and the highest foveal bulge point The red cross shows the center of the foveal avascular zone (FAZ) on the optical coherence tomography angiography (OCTA) image. The green and yellow crosses show the highest foveal bulge (FB) points that were measured manually using a B-scan image. The final highest FB point is defined as the center of the two measured highest FB points. The distance between the center of the FAZ and the highest FB point is measured (Blue arrow). 